\title{
Semantic Knowledge Transformation for Context-aware Heterogeneous Formalisms
}

\author{
Hafiz Mahfooz Ul Haque ${ }^{1}$ \\ Department of Software Engineering, \\ The University of Lahore, \\ Lahore, Pakistan
}

\author{
Sajid Ullah Khan ${ }^{2}$ \\ Department of Computer Science, \\ The University of Lahore, \\ Lahore, Pakistan
}

\author{
Ibrar Hussain ${ }^{3}$ \\ Department of Software Engineering, \\ The University of Lahore, \\ Lahore, Pakistan
}

\begin{abstract}
In recent years, an increasing social dependency has been observed over the cell phones and now evolved into smart devices. Due to the rapid escalation of these smart devices, users are becoming habitual in utilizing these services using smartphones and /or wearable devices in which different applications are running to assist and facilitate users in daily life routine activities. Mobility and context-awareness are the core features of pervasive computing. Context-awareness has the capability to identify the current situation and respond accordingly in the environment whenever and wherever needed. However, it is quite challenging to detect and sense the more appropriate contextual information when various interactive devices communicate among themselves. This paper presents the semantic knowledge transformation techniques for ontology-driven context-aware formalisms to model heterogeneous systems. We propose theoretical as well as practical approaches to transform semantic knowledge into firstorder Horn-clause rules format which can be used by contextaware multi-agent systems to achieve their desired goals.
\end{abstract}

Keywords-Context-aware system; semantic knowledge transformation; ontology; interoperability; smart spaces

\section{INTRODUCTION}

Smart computing has been considered as one of the most promising research area and rapidly evolving field around the globe. Since the last decade, smart computing has a remarkable upsurge and is continuously progressing towards the development of smarter, faster and tiny devices. Smartphones facilitate the users in daily routine tasks as well as enable interoperability to other active users in the same environment [1]. Smart device dependent technology helps to generate information about user's location, time, and movement to sense and can get a perception about the environment. Usually, knowledge engineers and context-aware computing systems e.g. multiple interacting smart devices and/or multi-agent systems are more user-oriented and formally specified approaches to model context-aware systems. Smart devices need machineoriented knowledge source intended to make it possible as an intelligent mechanism and smart decisions to perform in a flexible and adjustable way. This trend has been briskly advancing towards pervasive computing environment where smart devices are becoming intelligent enough to analyze the user's current situation and take decision according to the awareness level of the users, which is known as contextawareness. In a pervasive computing environment, semantic knowledge has gained a significant attention for modelling and reasoning heterogeneous systems using context-aware devices. It has been observed that from an individual perspective, a user does not hold the diverse kind of individualized data that can be obtained from heterogeneous sources with their usage pattern, however, it does not disturb their decision on acting [2]. Knowledge representation techniques play vital roles in context-aware computing for understanding contextual information, interpret them, act as a decision support system and then store contextualized information in the knowledge base. Literature has revealed various knowledge representation techniques such as semantic ontologies, frames, rule-based knowledge, knowledge map, decision tables and decision trees [3], [4] etc. Some of them are more useful for human understandability such as semantic knowledge so-called ontologies while some are more suitable for machine requirements for automated inferencing and reasoning process such as rulebased system and object-oriented knowledge representations.

In the literature, several approaches have been proposed using different algorithms [3], [5], transformation functions [6] to improve knowledge representation techniques. It helps knowledge workers to get intrude input knowledge into the system with better machine interoperability. In view of the analysis of the state-of-the-art research approaches, there are few controversial issues and flaws in the conversion of OWL constructs into different desirable knowledge sources [7] as knowledge may exist in different domains in a heterogeneous environment. It may involve inter-agent coordination in distributed nature. Knowledge is conceptualized from the diverse domain, so the flaws or challenging issues might be categorized in different preferences to be adapted. Few of the major challenges are the ambiguity of knowledge, level of automation, the ratio of human intervention \& machine intervention to the system, querying efficiency, reasoning \& inferring, complex computation and the scope of usability etc. Content-awareness is an appropriate paradigm to process and transmit data in different ways to communicate the content to the end-user. In this paper, we propose semantic knowledge transformation approaches to facilitate the user by introducing a well-defined model and reduce the gap between knowledge engineers and machine users to enhance knowledge interoperability.

The rest of the paper is organized as follows. In Section II, we briefly discuss the background knowledge of context, context-aware systems, contextualizing semantic knowledge and related work. Section III presents a theoretical mapping of ontology axioms to defeasible logic programming rules (DeLP). In Section IV, we show semantic knowledge transformation into a heterogeneous object text file (HOTF). In Section V, we present a contextual defeasible reasoning-based formalism for heterogeneous systems, and finally conclude in 
Section VI.

\section{PReliminaries AND Related Work}

\section{A. Context and Context-aware Systems}

Literature has revealed several definitions of context so far. The term context can be described as a set of occurring situations essential to the surrounding environment. The situations include in a context setting are persons, objects and the computing resources in the same environment. The ability of smart devices and smart applications to recognize existing working situations or context and adapt their behavior accordingly is known as context awareness [8].

Abowd et al. [9] define context as: "Context is any information that can be used to characterize the situation of an entity. An entity is a person, place, or object that is considered relevant to the interaction between a user and an application, including the user and applications themselves". In a pervasive computing environment, contextual information is of different kinds such as time-context, user-context, physical and computing context. Moreover, the detail about a specific user can be obtained as user profiles, user's location and social situation [10]. More specifically, context provides information related to an entity to know about its current status. It conveys knowledge about a user and application to be interlinked in a specific manner [11]. These devices used for human interactivity e.g. personal digital assistance devices, smart devices or mobile phones, and wireless sensor nodes almost have complex activities to be adapted. Sensors are used to sense the contextual information from the environment which is obtained by the acquisition modules in the context-aware systems. The data generated by the sensors is dynamic and the acquired contextual information can be used to perform reasoning to infer high-level context. The context is interpreted with respect to their scope and describes the context situation at a highlevel of interpretation. It is then presented to infer implications and the adaptation process facilitates the users in diverse routine activities manipulating decision-making techniques. The appropriated user's services are discovered and selected in the adaptation process and delivered to the user through the participant devices. The service behavior and the device behavior are adapted with the help of context data accordingly. The application domain is the smart spaces where users interact or communicate smart mobile devices e.g. homes, offices, community areas, healthcare, hotels, campuses and military places [2]. Context-aware approaches should be adaptable as per the changing situation so that user can perfectly complete their desired tasks across the physical locations and smart devices [12].

In the literature, much research efforts have been made to improve interoperability, balancing roles, collaborative work environment among machines and crowd sensing intelligence for human and computing processes design human in the loop architecture [13]. For multiple interacting devices, an agent can be programmed to solve the specific problem. In the multi-agent system, each agent has dedicated a role to capture the knowledge, analyze the situation based on its knowledge base, plan a sequence of actions to achieve the desired goal, enable interoperability in an efficient way, and then finally take decisions dynamically [14]. According to [15] the designing and modeling roles in agent-based systems to facilitate positive interactivity may provide better understandability to the knowledge workers, designer, and development staff. Semantically enriched ontologies are used as a naturally interactive mode among computing devices and active users are shown to be accurate choices [16]. An interoperability refers to the ability to analyze roles in common and to develop schemas in the deployment perspective realizing different methods and tactics. Knowledge interoperability can be further enhanced to cater to complex heterogeneous environments.

\section{B. Contextualizing Heterogeneous Knowledge Sources}

Recent years have witnessed the rapid advances in the field of semantic web. The semantic web is a set of standards that can be used for RDF data model, query languages and web ontology language (OWL) to store vocabularies and ontologies. Ontology is an explicit conceptualization of the domain having classes/concepts with their pre-defined relationships [17]. Literature has revealed two different versions of OWL (web ontology language) named as OWL 1 and OWL 2 each having their own sub-languages. A set of fuzzy variables are used for the contextual information and require the rules of fuzzy logic. Local storage stores the collected context data in encrypted form. A history of context is stored to identify preferences. An adequate memory requirement and firm data retrieval mechanisms are required for the context data to achieve efficiency. The missing variables values of the contextual data can be calculated through probabilistic techniques [2].

In literature, researchers have significant contribution in modeling heterogeneous systems by conjoining ontologies with various knowledge representation languages. Description logic with ontology mapping is the most appropriate knowledge representation technique. Distributed description logic (DDL) is a knowledge representation formalism that supports heterogeneity of information. It combines DL knowledge bases to represent distributed information. It also enables interontology mappings using distributed description logics [20]. According to [18], a DDL is mainly a generalization of the description logic frameworks. It aims to conceptualize multiple ontologies that are interconnected by semantic mappings. The core intention of interconnecting ontology axioms is to maintain their unique identity, independence, and preservation of individualized DL knowledge-bases. In DDL, multiple ontologies are interconnected to exchange information using semantic mapping [19] but the axioms of one ontology cannot be shared with other ontology. In DDL, each local ontology contains its local knowledge base with distributed reasoning. The knowledge base of each of the local ontology consists of two kinds of axioms; TBox and ABox. The correspondences of different ontology axioms are called bridge rules (or interontology axioms). By using bridge rules, TBox axioms of one ontology can be interlinked to the corresponding TBox axioms of another ontology in an implicit manner. Example of the inter-ontology axiom is bridge rule which is represented as $C_{i} \sqsubseteq D_{j} ; C_{i} \sqsupseteq D_{j}$; Where $C_{i}$ and $D_{j}$ belong to concepts of ontologies $O_{i}$ and $O_{j}$ correspondingly. A group of different DL knowledge bases is the composition of distributed DL knowledge bases (DKB) that can be expressed as a pair ( $T$, $A$ ) that includes distributed TBoxes (DTBox) and distributed 
ABoxes (DAbox). For context modeling, ontology-based context modeling approach has been considered to be the most promising one [21].

\section{Related Work}

In recent years, context-aware applications are rapidly evolving and literature shows the substantial volume of research efforts with the incorporation of heterogeneous systems. Different approaches have been focused on and analyzed in this regard. In [5], semantic knowledge has been transformed into a desirable knowledge format. Authors proposed a transformation mechanism to convert semantic networks into frames. Semantic network ontologies are developed using Protégé ontology editor to get the OWL constructs. The frame is a knowledge representation technique which is more expressive in a sense that it allows the corporate manager or business analyst to predict the current trends in the specified domain. In light of the previous work [6] author has extended his research work and introduced semantic network as ontologies developed in protégé, defined transformation mechanism and provides frames structure as a result. A case study is adapted to show the working flow of the system and to validate the results. Totkov et al. [32] have proposed frame models in e-learning. It introduces the significance of frames and their implications in different real-world application domains such as such as Elearning, which is fanatical to the notion of frames usability as a significant knowledge representation technique. Keeping in view the significance of semantic knowledge transformation into a generalized format for automated reasoning of largescale ontology management, a mapping of OWL constructs is introduced into relational database schema [33]. It performs the corresponding mapping of classes, properties and individual instances of ontology to the database schema. They use SPARQL and SQL query to query corresponding axioms in ontology and database instances respectively. In [34], an OWLAPI based translator, Onto-HCR, was proposed to translate the ontology domain into a set of the plain text of Horn-clause rules format. Later in [35], authors proposed the enhanced version of the tool, D-Onto-HCR. This is also an OWL-API based translator which extracts rules from multiple ontology domains and then transform into horn-clause rules format. It gets input as ontologies and then translates the set of axioms into a set of plain text Horn-clause rules. Apart from this, this tool can be used to generate bridge rules. This work has been further enhanced by the same author in [22]. A translator tool, EDOH was developed which is an extended version of D-Onto-HCR. It is capable to transform mapping rules which help the context-aware defeasible reasoning- based frameworks to deal with inconsistent and incomplete behavior of the system. It takes OWL 2 RL ontology as an input file which is a readable file source using Protégé in the OWL/XML format and provides the resulted output as a text file. In contrast to previous work, the semantic knowledge transformation approaches proposed in this paper provide more flexibility in terms of acquiring contextual information from semantic knowledge sources. We chose the ontology-based context modeling approach and develop the ontologies of smart rescue system and a medical diagnostic system given in the following sections.

\section{Transforming Ontology Axioms into DeLP RULES}

In this section, we theoretically transform semantic knowledge into horn-clause rules format following the mapping of ontology to DL knowledge-base and then defeasible logic programming (DeLP). To suitably model the system using multiple ontologies and transforming heterogeneous semantic knowledge into defeasible logic programming rules, we adopt the case study of smart rescue system from [22] to illustrate the expressivity and efficacy of the system. We develop two different ontologies of smart rescue system to monitor the fear of flying people and elderly people to provide suitable cure at the right time and in the right place. Fragments of these ontologies can be seen in Fig. 1 and in Fig. 2.

To model the domains, we construct OWL 2 RL ontology (sub-language of OWL 2 [23] to model the case study due to its scalable rule-based reasoning system and expressive power. We show the expressivity and dynamicity of the system by constructing more complex rules using SWRL (Semantic web rule language) [24] in Protégé ontology editor [25]. SWRL provides privileges to users to construct rules using unary predicates (OWL concepts) and binary predicates (roles). In this paper, we explicitly conjoin OWL 2 RL with SWRL rules to solve complex problems using semantic context modeling approach. Contextual knowledge in term of classes and relationships can be defined as concepts and roles respectively in the description logic. Description Logics (DLs) are based on ontology and it has been considered as the best-suited approach to model the domain using ontology with the powerful and expressive DL reasoning. DLs have different sub-languages, among others, we consider DL-SROIQ due to its expressive formalism and its inferencing is decidable [26], [27]. So, ontology axioms can be mapped with the corresponding DL syntax [28] which is shown in Table I. As DL can be used to model the domain, so we transform smart rescue system ontology axiom into corresponding DL axioms. DL Knowledge-base consists of terminology box (TBox) and assertion box (Abox). TBox represents the axioms whereas ABox depicts their substitutions. In Table II, we show the corresponding ontology axioms transformation into DL Knowledge-base.

Description Logic program (DLP) acts as an intermediary knowledge representation mechanism between description logic (DL) and logic programming [28]. DL is a subset of First Order logic (FOL), so DL axioms can be transformed into FOL. Logic programming is closely related to the Horn

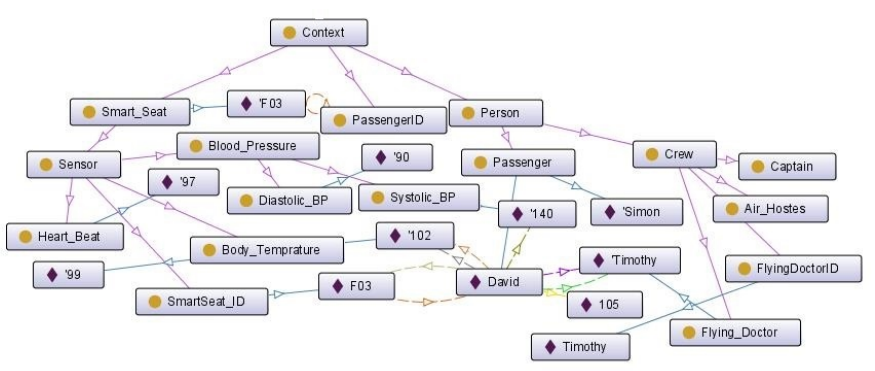

Fig. 1. Smart Seat Ontology Fragment 
TABLE I. OWL 2 RL AXIOM TO DL AXIOM

\begin{tabular}{|c|c|}
\hline OWL 2 RL Axiom & DL Axiom \\
\hline SubClassOf & $C \sqsubseteq D$ \\
SubObjectPropertyOf & $R \sqsubseteq S$ \\
EquivalentClassOf & $C \equiv D$ \\
EquivalentObjectPropertyOf & $R \equiv S$ \\
InverseObjectPropertyOf & $R \equiv S^{-}$ \\
ObjectPropertyChain & $R \circ S \sqsubseteq S$ \\
ObjectPropertyRange & $\top \sqsubseteq \forall \bar{R} . C$ \\
ObjectPropertyDomain & $\top \sqsubseteq \forall R^{-} . C$ \\
SymmetricObjectProperty & $R \equiv R^{-}$ \\
ObjectSomeValuesFrom & $\exists R . C \sqsubseteq D$ \\
ObjectAllValuesFrom & $C \sqsubseteq \forall R . D$ \\
\hline
\end{tabular}

fragment of FOL but it is not expressible using FOL [29]. Moreover, DeLP [30] has been considered as one of the most promising approaches to translate DL axioms into nonmonotonic horn-clause rules. As this framework is distributed in nature and each domain has to retain its independence, uniqueness, preservation of identity and security. So the acquisition of contextual knowledge from different ontologies and constructing rules could cause inconsistency. To suitably handle this situation, we transform DL knowledge into DeLP rules in the form of strict and defeasible rules as shown in Table III. Strict rules are non-contradictory and interpreted in a classical sense. Defeasible rules perform reasoning to defeat the conflicting contextual information.

\section{Semantic Knowledge To Heterogenous ObJect TEXT FILE (HOTF)}

This section presents a systematic tool based approach to transform the semantic knowledge into plain text of OWL constructs. We present a transformation mechanism using HOTF (Heterogeneous Object Text File). Context-aware agents acquire the ontology-driven contextual information from different ontologies using HOTF, perform reasoning and then adapt behavior accordingly. It is more expressive knowledge representation technique and this translation process is automated. The design of HOTF corresponds to the ontology structural specification and hence there is a proximal oneto-one transformation from multiple ontologies constructs to heterogeneous object text file. We illustrate the working flow of HOTF using the case study of medical ontologies. This case study is constructed to assists the patients in diagnostic perspective and for doctors in the treatment plan and healthrelated services. This ontology is specifically designed to provide complete medical services to patients to take appropriate medical services and this system automatically generates alert messages to doctors whenever needed. We develop ontologies using Protégé [25] ontology editor and it can be saved in

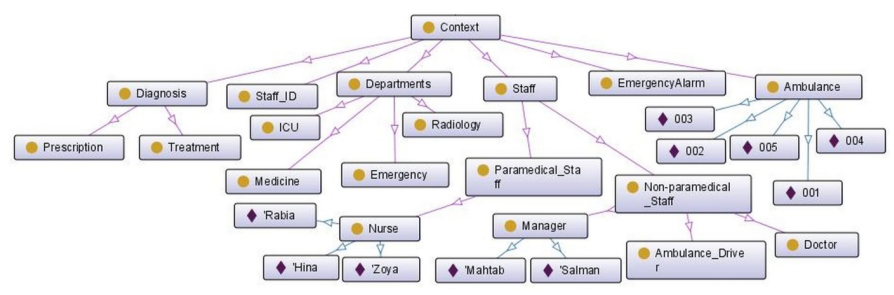

Fig. 2. Smart Rescue System Ontology Fragment

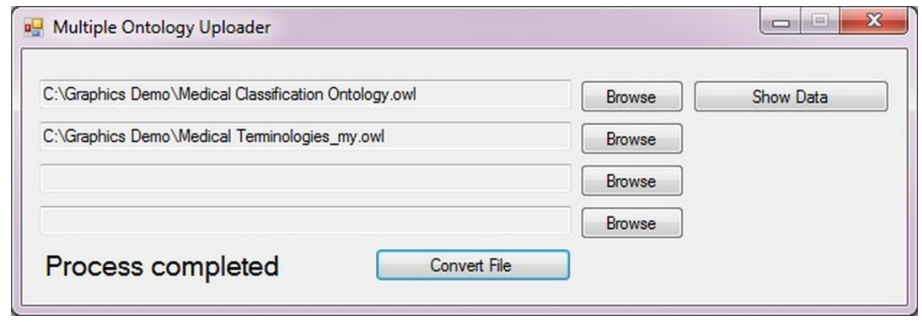

Fig. 3. Multiple Ontology Uploader

OWL/XML and/or OWL/RDF format. For the translation process, we upload multiple ontologies as input to HOTF as shown in Fig. 3.

Fig. 3 shows a window of multiple ontology uploader which is an input window to get the ontology from different domains of heterogeneous systems. After uploading files, the "Convert File" button executes the transformation function to transform ontological knowledge into Heterogeneous Object Text File. It also classifies OWL constructs based on their object properties and data properties. By clicking "Show Data" button, multiple ontologies OWL constructs can be seen in Fig. 4 in the form of class names with their predefined relationship among classes, methods and relationship types such as aggregation, association etc.

Using this approach, at the moment, four different ontologies can be converted into Object text file simultaneously. However, the number of ontologies can be increased based on user requirement. The results obtained using HOTF can be further manipulated to express in the form of horn-clause rules in order to provide better query efficiency. Apart from this, the medical case study discussed above can be interlinked with smart rescue system (mentioned in the previous section) using context-aware multi-agent reasoning based formalism. We draft the ontology-driven context-aware multi-interactive system in Fig. 5 to depict the transformation function from semantic knowledge into rule-based knowledge. The transformation from multiple ontologies to HOTF is implicit in nature; however, from HOTF to rule-based knowledge transformation can be done statically.

\section{Contextual Defeasible Reasoning Based FORMALISM FOR HETEROGENOUS SYSTEMS}

We present the contextual defeasible reasoning based multiagent formalism which uses the ontology-driven horn-clause rules to model heterogeneous system. Contextual defeasible reasoning has been emerged from defeasible logic and multicontext system. Contextual Defeasible Logic (CDL) is a rule-based reasoning technique which incorporates defeasible logic in order to defeat and/or derive the contrary evidence. Defeasible logic has been considered as one of the most promising approaches in non-monotonic reasoning. It is a simple and efficient reasoning technique which performs reasoning monotonically as well as non-monotonically [31]. CDL essentially consists of a set of rules (strict, defeasible and defeater), a set of vocabulary and preference ordering. There are two kinds of reasoning in CDL: local reasoning and global reasoning. Local reasoning is performed by either strict rules 
TABLE II. MAPPING FROM DL AXIOMS to STRICT AND DEFESIBLE TERMinologies
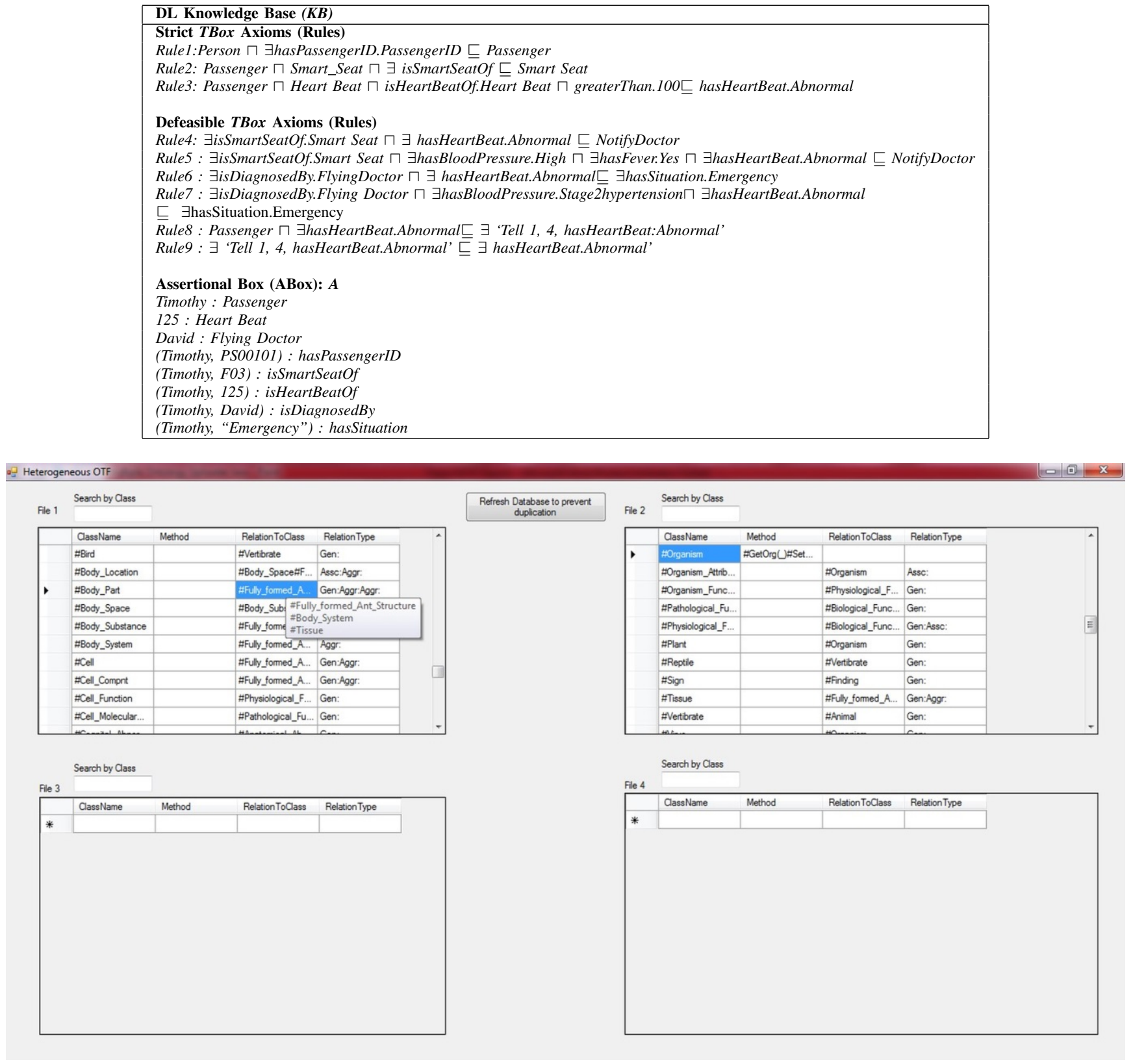

Fig. 4. Heterogenous OTF (HOTF)

or defeasible rules or a combination of both, and these rules are extracted from either a single-domain ontology or multidomain ontologies. Strict rules are interpreted for local domain ontology in a classical sense whereas defeasible rules are interpreted defeasibly. Global reasoning is performed by firing the rule instances of mapping rules. These rules are formed by combining contextual information extracted from different domains ontologies. More precisely, these rules are formed from local contexts of one domain with the global context of another domain in order to perform successful execution of the system. The set of individualized contextual information and the set of rules taken from corresponding ontology domain is known as local contexts whereas the contextual information extracted from another ontology is known as foreign contexts. These set of local and foreign contexts are formed to make the bridge rules and these rules are interpreted in a classical way using context-aware defeasible reasoning based multi-agent system.

This system includes a set of agents $n_{A_{g}}(\geq 1)$, i.e., $A_{g}=\left\{1,2, \ldots, n_{A_{g}}\right\}$. Each agent is assigned a specific task to solve the problem and it is dedicated for its own specified tasks only. Each agent $\left(i \in A_{g}\right)$ in the system having of a set of rules (strict, defeasible, mapping and communication rules) along with the set of contextual information (vocabulary) and 
TABLE III. MAPPING FROM STRICT AND DEFEASIBLE TERMINOLOGIES INTO HORN-CLAUSE RULES

\begin{tabular}{l}
\hline DL Axioms to Rules Mapping \\
\hline Strict TBox Axioms to Strict Rules Mapping \\
Rule1: Person(?pas), hasPassengerID(?pas, ?pasID), PassengerID(pasID) \\
$\rightarrow$ Passenger(?pas) \\
Rule2: Passenger(?pas), Smart_Seat(?sid) $\rightarrow$ isSmartSeatOf (?p, ?sid) \\
Rule3: Passenger(?pas), isHeartBeatOf (?hb, ?pas), HeartBeat(?hb), \\
greaterThan(?bh, 100) $\rightarrow$ hasHeartBeat(?pas, "abnormal") \\
\\
Defeasible TBox Axioms to Defeasible Rules Mapping \\
Rule4: isSmartSeatOf (?sid, ?pas), hasHeartBeat(?pas, "Abnormal") \\
$\Rightarrow$ NotifyDoctor(?p, ?sid) \\
Rule5: isSmartSeatOf (?sid, ?pas), hasBloodPressure(?pas, "High"), \\
hasFever(?p, "Yes"), hasHeartBeat(?p, "Abnormal") $\Rightarrow$ NotifyDoctor(?pas, ?sid) \\
Rule6: isDiagnosedBy(?p, ?doc), hasHeartBeat(?p, "Abnormal") \\
$\Rightarrow$ hasSituation(?p, "Emergency") \\
Rule7: isDiagnosedBy(?p, ?doc), hasBloodPressure(?p, "Stage2hypertension"), \\
hasHeartBeat(?p, "Abnormal") $\Rightarrow$ hasSituation(?p, "Emergency") \\
Communication Rules \\
Rule8: Passenger(?pas), hasHeartBeat(?pas, "Abnormal") \\
$\rightarrow$ 'Tell 1, 4, hasHeartBeat'(?pas, "Abnormal") \\
Rule9: 'Tell 1, 4, hasHeartBeat'(?pas, "Abnormal') \\
$\rightarrow$ hasHeartBeat(?pas, "Abnormal")
\end{tabular}

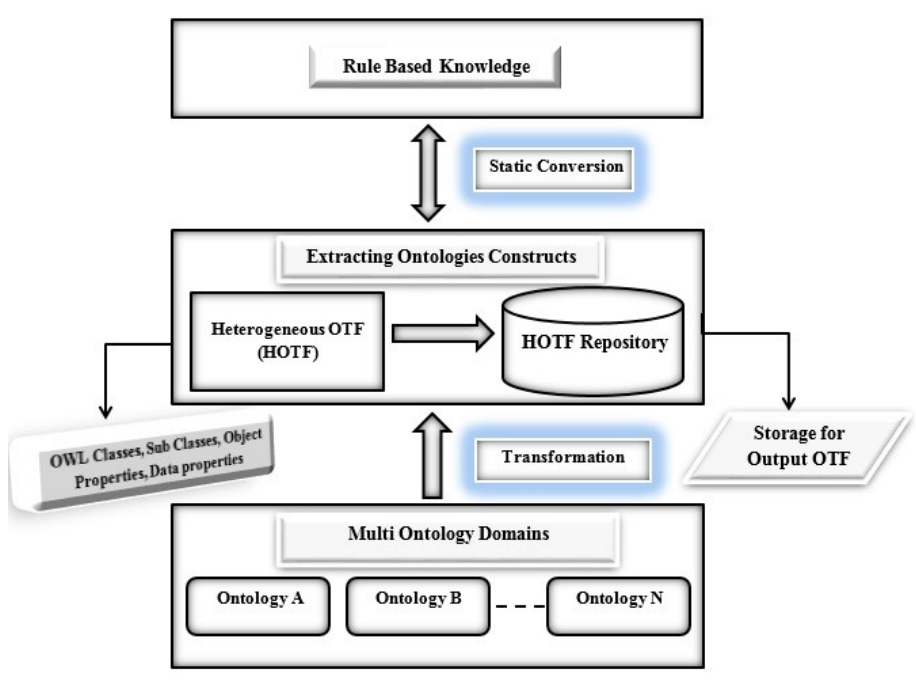

Fig. 5. Ontology-Driven Context-Aware Multi-Interactive System

priority relation, which is represented using a triple $(R, F,>)$, where $F$ is a finite set of individualized contextual information (facts), $R$ represents the set of all rules which are used to infer derivated contextualized information from heterogeneous knowledge sources, and $>$ express superiority relation explicitly on $R$. As the system is heterogeneous in nature and runs in a highly decentralized and dynamic environment, so agents need to derive and share contextual information autonomously. For contextual modeling and reasoning, the system performs three core actions: Rule, Copy and Idle. Rule action is triggered by firing the rule instances of each agent in the system. Rule instances are of the type of strict rules, defeasible rules, and mapping rules. Copy action is triggered by firing the instance of communication rules whenever agents need to exchange the contextual information. Idle action allows agents to remain in idle state but transit to the next state if triggered by the system. The system performs reasoning non-deterministically and non-monotonically, so the rule priorities are static and set at the design time of the system in order to avoid inconsistent behavior of the system.

To illustrate the use of the proposed formalism, we develop the ontologies of smart rescue system and smart seat system to assist the patient's diagnostic perspective and to monitor the vital signs of the fear of flying people during their air travel. We omit the technical details of contextual defeasible reasoning framework in this paper. For this, we refer the interested readers to our earlier work [22]. However, in Section III, we have discussed the implementation of the extended case study in terms of rules transformation.

\section{CONCLUSiOn AND Future Work}

In this paper, we proposed two approaches of semantic knowledge transformation. The first approach provides a concrete and proximal one-to-one transformation mechanism from ontology axiom to DeLP rules. The second approach provides an automated transformation of ontology axioms into contextaware agent's understandable format in HOTF so that agents automatically acquire contextual information from HOTF to model heterogeneous system and thus achieve their desired goals. In the future, we will develop a systematic application model and implement the case study to check, specify and verify its correctness properties of the system.

\section{REFERENCES}

[1] R. Ballagas, J. Borchers, M. Rohs and J. G. Sheridan, The smart phone: a ubiquitous input device, in IEEE Pervasive Computing, vol. 5, no. 1, Jan.-March 2006, pp. 70-77.

[2] Grabowski, P., \& Samfelt, J. (2016). User awareness of privacy regarding user data in Mobile Health applications and wearables:-Do you know what you are sharing?

[3] Liao, S. H. (2005). Expert system methodologies and applications, a decade review from 1995 to 2004. Expert systems with applications, 28(1), 93-103.

[4] J. D. Wren, Engineering in genomics, in IEEE Engineering in Medicine and Biology Magazine, pp. 87-93, (2004).

[5] Khan, S. U. (2013). Transformation of Semantic Networks into Frames. International Journal of Innovation, Management and Technology, 4(1), 21.

[6] Khan, S. U., Khan, M., \& Nauman, M. (2016, August). Semi-automatic knowledge transformation of semantic network ontologies into Frames structures. In Innovative Computing Technology (INTECH), 2016 Sixth International Conference on (pp. 712-717). IEEE.

[7] Afzal, H., Waqas, M., \& Naz, T. (2016). OWLMap: Fully Automatic Mapping of Ontology into Relational Database Schema. International journal of advanced computer science and applications, 7(11), 7-15.

[8] Dey, A. K. (2001). Understanding and using context. Personal and ubiquitous computing, 5(1), 4-7.

[9] Abowd, G. D., Dey, A. K., Brown, P. J., Davies, N., Smith, M., \& Steggles, P. (1999, September). Towards a better understanding of context and context-awareness. In International Symposium on handheld and ubiquitous computing (pp. 304-307). Springer, Berlin, Heidelberg.

[10] Schilit, B., Adams, N \& Want, R. (1994, December). Context-aware computing applications. In Mobile Computing Systems and Applications, 1994. Proceedings., Workshop on (pp. 85-90). IEEE.

[11] Guo, B., Zhang, D., \& Imai, M. (2011). Toward a cooperative programming framework for context-aware applications. Personal and ubiquitous computing, 15(3), 221-233.

[12] Li, H., \& Wang, J. (2011, August). Application architecture for ambient intelligence systems based on context ontology modeling. In Internet Technology and Applications (iTAP), 2011 International Conference on (pp. 1-4). IEEE. 
[13] Nunes, D. S., Zhang, P., \& Silva, J. S. (2015). A survey on human-inthe-loop applications towards an internet of all. IEEE Communications Surveys \& Tutorials, 17(2), 944-965.

[14] Wooldridge, M. (2009). An introduction to multiagent systems. John Wiley \& Sons.

[15] Cabri, G., Ferrari, L., \& Leonardi, L. (2004, October). Agent role-based collaboration and coordination: a survey about existing approaches. In Systems, Man and Cybernetics, 2004 IEEE International Conference on (Vol. 6, pp. 5473-5478). IEEE.

[16] Soylu, A., Giese, M., Jimenez-Ruiz, E., Vega-Gorgojo, G., \& Horrocks, I. (2016). Experiencing OptiqueVQS: a multi-paradigm and ontologybased visual query system for end users. Universal Access in the Information Society, 15(1), 129-152.

[17] Guarino, N., Oberle, D., \& Staab, S. (2009). What is an ontology?. In Handbook on ontologies (pp. 1-17). Springer, Berlin, Heidelberg.

[18] Serafini, L., \& Tamilin, A. (2005, May). Drago: Distributed reasoning architecture for the semantic web. In European Semantic Web Conference (pp. 361-376). Springer, Berlin, Heidelberg.

[19] Grau, B. C., Parsia, B., \& Sirin, E. (2004, November). Working with multiple ontologies on the semantic web. In International Semantic Web Conference (pp. 620-634). Springer, Berlin, Heidelberg.

[20] Borgida, A., \& Serafini, L. (2002, October). Distributed description logics: Directed domain correspondences in federated information sources. In OTM Confederated International Conferences" On the Move to Meaningful Internet Systems" (pp. 36-53). Springer, Berlin, Heidelberg.

[21] Baldauf, M., Dustdar, S., \& Rosenberg, F. (2007). A survey on contextaware systems. International Journal of Ad Hoc and Ubiquitous Computing, 2(4), 263-277.

[22] Haque, H. M. U., \& Khan, S. U. A context-aware reasoning framework for heterogeneous systems. In International Conference on Advancements in Computational Sciences (ICACS'18), IEEE Xplore Digital Library, pp. 1-9, (2018).

[23] Hitzler, P., Krötzsch, M., Parsia, B., Patel-Schneider, P. F., \& Rudolph, S. (2009). OWL 2 web ontology language primer. W3C recommendation, 27(1), 123.
[24] O‘connor, M., Knublauch, H., Tu, S., \& Musen, M. (2005). Writing rules for the semantic web using SWRL and Jess. Protégé with Rules WS, Madrid.

[25] Protégé: The Protégé ontology editor and knowledge-based framework (Version 4.1). http://protege.stanford.edu/ (July 2011)

[26] Horrocks, I., Kutz, O., \& Sattler, U. (2006), The Even More Irresistible SROIQ. Kr, 6, 57-67.

[27] O'Connor, M. J. \& Das, A. K. (2012), A Pair of OWL 2 RL Reasoners. In OWLED (Vol. 849).

[28] Grosof, B. N., Horrocks, I., Volz, R., \& Decker, S. (2003, May). Description logic programs: combining logic programs with description logic. In Proceedings of the 12th international conference on World Wide Web (pp. 48-57). ACM.

[29] Baral, C. and Gelfond, M. Logic programming and knowledge representation. The Journal of Logic Programming, 19:73148, (1994).

[30] Gómez, S. A., Chesnevar, C. I., \& Simari, G. R. (2007). Inconsistent ontology handling by translating description logics into defeasible logic programming. Inteligencia Artificial. Revista Iberoamericana de Inteligencia Artificial, 11(35).

[31] Antoniou, G. (2002). A Nonmonotonic Rule System using Ontologies. In RuleML (Vol. 60).

[32] Totkov G., S. Gaftandzhieva, R. Doneva, Accumulative frame models in e-learning, Scientific works of the Union of Scientists in Bulgaria. Plovdiv, Series C. Technik and Technologies. ISSN 1311-9419, Vol. 15, pp. 17-20, 2017.

[33] Lee, J., Goodwin, R., \& Akkiraju, R. (2006). Ontology management for large-scale enterprise systems. In Web Semantics \& Ontology (pp. 91-114). IGI Global.

[34] Ul-Haque, H. M. (2017). A formal approach to modelling and verification of context-aware systems (Doctoral dissertation, University of Nottingham).

[35] Haque, H. M. U., Rakib, A., \& Uddin, I. (2016, November). Modelling and reasoning about context-aware agents over heterogeneous knowledge sources. In International conference on context-aware systems and applications (pp. 1-11). Springer, Cham. 